\title{
ON A THEOREM OF P. L. ULJANOV
}

VERA B. STANOJEVIC

\begin{abstract}
It is shown that if $c(n)=o(1),|n| \rightarrow \infty$, and $\sum_{|n|<x}\left|J^{\prime \prime \prime} c(n)\right|<x$, for some integer $m \geqslant 1$, then the series $\sum_{|n|<\infty} c(n) e^{i n t}$ converges to some $f \in I^{\prime \prime}(\mathbf{T})$ for any $0<p<1 / m$.
\end{abstract}

As an application of $A$-integrals, P. L. Uljanov [1] obtained an interesting result concerning the integrability of $|f|^{p}$ and $|\bar{f}|^{p}$, for any $0<p<1$, where

$$
\begin{gathered}
f(x)=\sum_{k=1}^{\infty} a_{k} \cos k x, \\
\bar{f}(x)=\sum_{k=1}^{\infty} a_{k} \sin k x
\end{gathered}
$$

and $\left\{a_{n}\right\}$ is a null-sequence of bounded variation.

TheOREM (P. L. UlJanov [1]). Let $\left\{a_{n}\right\}$ be a null-sequence of bounded variation. Then for any $0<p<1,|f|^{p}$ and $|\bar{f}|^{p}$ are integrable, where $f$ and $\bar{f}$ are defined by (1) and (2).

In this paper I shall prove a version of Uljanov's theorem and extend it to the series

$$
\sum_{|n|<\infty} c(n) e^{i n t}, \quad t \in \mathbf{T}=\mathbf{R} / 2 \pi \mathbf{Z}
$$

where $\{c(n)\}_{|n|<\infty}$ is a null-sequence of complex numbers such that for some integer $m \geqslant 1$

$$
\sum_{|n|<\infty}\left|\Delta^{m} c(n)\right|<\infty
$$

where $\Delta^{m} c(n)=\Delta^{m-1} c(n)-\Delta^{m-1} c(n+1)$. J. W. Garrett, C. S. Rees and Č. V. Stanojevic [2] considered (4) in the case of real sequences. They also gave an example in [2] to show that (4) is an effective generalization of sequences of bounded variation.

In this paper I shall establish the following result.

Received by the editors April 20, 1983.

1980 Mathematics Subject Classification. Primary 42A20, 42 A32.

Kev words and phrases. Convergence in $L^{p}(\mathbf{T})$-metric $(0<p<1)$ of trigonometric series.

$$
\begin{aligned}
& \text { 1984 American Mathematical Society } \\
& 0002-9939 / 84 \$ 1.00+\$ .25 \text { per page }
\end{aligned}
$$


THEOREM. Let $\{c(n)\}_{|n|<x}$ be a null-sequence of complex numbers. If, for some integer $m \geqslant 1,(4)$ holds, then the point-wise limit $f$ of

$$
S_{n}(t)=\sum_{|,| \leqslant n} c(j) e^{i, j t}
$$

exists in $\mathbf{T}-\{0\}$ and for any $0<p<1 / m,|f|^{p}$ and $|\bar{f}|^{p}$ are integrable.

Proof. Let $m \geqslant 1$ and let $M<N$. Define

$$
A_{M, N+1}^{(k)}=A_{M . N+1}^{(k)}(t)=\left[\Delta^{k} c(M)\right] e^{i M t}-\left[\Delta^{k} c(N+1)\right] e^{i(N+1) t}
$$

and $w=w(t)=1-e^{-i t}$. Then the following identity can be established:

$$
w^{m} \sum_{j=M+m}^{N+m} c(j) e^{i j t}=\sum_{j=M}^{N}\left[\Delta^{m} c(j)\right] e^{i j t}-\sum_{k=0}^{m} w^{k} A_{M+k, N+h \cdot 1}^{(m-1) k)}
$$

for any sequence $\{c(n)\}_{|n|<x}$ of complex numbers. Consequently, setting $M=-n$, $N=n$, for $t \neq 0$ we have

$$
\begin{aligned}
S_{n}(t)= & \frac{1}{w^{m}} \sum_{|j| \leqslant n}\left[\Delta^{m} c(j)\right] e^{i j t}-\frac{1}{w^{m}} \sum_{k=0}^{m-1} w^{k} A_{-k, n+k+1}^{(m-1) k)} \\
& +\sum_{j=-n}^{-n+m-1} c(j) e^{i j t}-\sum_{j=n+1}^{n+m} c(j) e^{i j t} .
\end{aligned}
$$

Since (4) holds, the first term on the right-hand side of (6) converges point-wise in $\mathbf{T}-\{0\}$. The rest of the terms tend to zero because $c(n)=o(1),|n| \rightarrow \infty$. Hence the limit of $S_{n}(t), n \rightarrow \infty$, exists in $\mathbf{T}-\{0\}$ and we denote it by $f$.

It remains to prove that for any $0<p<1 / m,|f| \in L^{p}(\mathbf{T})$. Since $S_{n}(t)$ is a trigonometric polynomial it suffices to show that $S_{n}(t)$ converges to $f$ in $L^{p}(\mathbf{T})$ metric for any $0<p<1 / m$. For $t \neq 0$ consider

$$
\begin{aligned}
f(t)-S_{n}(t)= & \frac{1}{w^{m}} \sum_{|n|<x}\left[\Delta^{m} c(n)\right] e^{i n t} \\
& -\frac{1}{w^{m}} \sum_{|j| \leqslant n}\left[\Delta^{m} c(j)\right] e^{i j t}+\frac{1}{w^{m}} \sum_{k=0}^{m-1} w^{k} A_{-n+k, n+k+1}^{(m-1-k)} \\
& -\sum_{j=-n}^{-n+m-1} c(j) e^{i j t}+\sum_{j=n+1}^{n+m} c(j) e^{i j t} .
\end{aligned}
$$

Hence we have

$$
\begin{aligned}
f(t)-S_{n}(t)= & \frac{1}{w^{m}} \sum_{|j| \geqslant n+1}\left[\Delta^{m} c(j)\right] e^{i j t}+\frac{1}{w^{m}} \sum_{k=0}^{m-1} w^{k} A_{-n+k, n+k+1}^{(m-1-k)} \\
& -\sum_{j=-n}^{-n+m-1} c(j) e^{i j t}+\sum_{j=n+1}^{n+m} c(j) e^{i j t} .
\end{aligned}
$$


For $0<p<1 / m$ we obtain

$$
\begin{aligned}
\left|f(t)-S_{n}(t)\right|^{p} & \leqslant \frac{1}{|w(t)|^{m \cdot p}}\left(\sum_{|, j| \geqslant n+1}\left|\Delta^{m} c(j)\right|\right)^{p} \\
& +\frac{2^{m-1}}{|w(t)|^{m p}}\left(\sum_{k=0}^{m-1}\left(\left|\Delta^{(m-1-k)} c(-n+k)\right|+\left|\Delta^{(m-1-k)} c(n+k+1)\right|\right)\right)^{p} \\
& +\left(\sum_{j=-n}^{-n+m-1}|c(j)|\right)^{p}+\left(\sum_{j=n+1}^{n+m}|c(j)|\right)^{p} .
\end{aligned}
$$

Since for $m p<1$

$$
\int_{\mathbf{T}} \frac{d t}{|w(t)|^{m p}}<K
$$

where $K$ is an absolute constant, we have

$$
\begin{aligned}
\int_{\mathbf{T}}\left|f(t)-S_{n}(t)\right|^{p} d t \leqslant K\left(\sum_{|j| \geqslant n+1}\left|\Delta^{m} c(j)\right|\right)^{p} \\
+2^{m-1} K\left(\sum_{k=0}^{m-1}\left(\left|\Delta^{(m-1-k)} c(-n+k)\right|+\left|\Delta^{(m-1-k)} c(n+k+1)\right|\right)\right)^{p} \\
+m^{p} \max _{-n \leqslant j \leqslant-n+m-1}|c(j)|^{p}+m^{p} \max _{n+1 \leqslant j \leqslant n+m}|c(j)|^{p} .
\end{aligned}
$$

The second sum on the right-hand side of the last inequality is a finite sum of $o(1)$ terms, as $n \rightarrow \infty$, and the last two terms are trivially $o(1)$. Hence

$$
\left(\int_{\mathbf{T}}\left|f(t)-S_{n}(t)\right|^{p} d t\right)^{1 / p}=O\left(\sum_{|j| \geqslant n+1}\left|\Delta^{m} c(j)\right|\right), \quad n \rightarrow \infty .
$$

Finally, because of (4),

$$
\left(\int_{\mathbf{T}}\left|f(t)-S_{n}(t)\right|^{p} d t\right)^{1 / p}=o(1), \quad n \rightarrow \infty .
$$

Thus $|f| \in L^{p}(\mathbf{T})$ for any $0<p<1 / m$. That concludes the proof of the Theorem.

In a similar manner we can obtain that $|\bar{f}| \in L^{p}(\mathbf{T})$ for any $0<p<1 / m$. For $m=1$ we have the theorem of P. L. Uljanov.

The case $m=2$ is related to some subtle questions about $H^{p}$-spaces and it will be studied in a subsequent paper.

\section{REFERENCES}

1. P. L. Uljanov, Application of A-integration on a class of trigonometric series, Mat. Sb. 35 (77) (1954), 469-490. (Russian)

2. J. W. Garrett, C. S. Rees and Č. V. Stanojevic, $L^{1}$-convergence of Fourier series with coefficients of bounded variation, Proc. Amer. Math. Soc. 80 (1980), 423-430.

Department of Mathematics and Statistics, University of Missouri - Rolla, Rolla, Missouri 65401 